Fermilab-Conf-09-646-APC

December 2009

\title{
PION PRODUCTION FOR NEUTRINO FACTORIES AND MUON COLLIDERS ${ }^{* \dagger}$
}

\author{
N.V. MOKHOV, K.K. GUDIMA, J.B. STRAIT, S.I. STRIGANOV \\ Fermilab,MS 220, P.O. Box 500 \\ Batavia, IL 60510, U.S.A.
}

\begin{abstract}
Optimization of pion and muon production/collection for neutrino factories and muon colliders is described along with recent developments of the MARS15 code event generators and effects influencing the choice of the optimal beam energy.
\end{abstract}

\footnotetext{
"Work supported by Fermi Research Alliance, LLC under contract No. DE-AC02-07CH11359 with the U.S. Department of Energy.

'Presented paper at Workshop on Applications of High Intensity Proton Accelerators, October 19-21, 2009, Batavia, Illinois
} 


\title{
PION PRODUCTION FOR NEUTRINO FACTORIES AND MUON COLLIDERS
}

\author{
N.V. MOKHOV ${ }^{\dagger}$, K.K. GUDIMA, J.B. STRAIT, S.I. STRIGANOV \\ Fermilab,MS 220, P.O. Box 500 \\ Batavia, IL 60510, U.S.A.
}

Optimization of pion and muon production/collection for neutrino factories and muon colliders is described along with recent developments of the MARS15 code event generators and effects influencing the choice of the optimal beam energy.

\section{Target System}

Neutrino factory and muon collider (NFMC) projects are feasible only if adequate numbers of muons can be produced and collected. In the baseline frontend, a proton beam hits a thick target in the center of a high-field solenoid, followed by a matching section and a solenoidal decay channel which collects muons resulting from pion decays. A list of targetry issues includes [1]: maximal production of pions resulting in the highest number of useful muons at the downstream end of the system, background particles transported down the beamline, target survivability, beam window fatique and structural integrity, superconducting (SC) coil quench stability, heat loads on target and cryogenic system, radiation damage and activation of materials near the beam, spent proton beam handling, and numerous shielding issues from prompt radiation to groundwater activation. "Useful" muons are those captured by a phase rotation system downstream of the decay channel, with a kinetic energy in the range $40<\mathrm{T}_{\mu}<$ $180 \mathrm{MeV}$.

All these issues have been addressed in detailed simulations. Thorough MARS optimizations of the target system have been performed $[1,2]$ for 4 to 30 $\mathrm{GeV}$ proton beams. The findings included: a gain from high- $\mathrm{Z}$ target materials, especially at the high beam energies; a 1-m long hybrid solenoid at $B_{z} \times R_{a}{ }^{2}=1125$ $\mathrm{T} \times \mathrm{cm}^{2}$ with $\mathrm{B}_{\mathrm{z}}=20 \mathrm{~T}$ and aperture $\mathrm{R}_{\mathrm{a}}=7.5 \mathrm{~cm}$ followed by a matching section (to $\sim 20 \mathrm{~m})$ and decay channel $(\mathrm{R}=30 \mathrm{~cm}, \mathrm{~B}=1.25 \mathrm{~T})$ to $50-100 \mathrm{~m}$ with $\mathrm{SC}$ coils

\footnotetext{
† E-mail: mokhov@fnal.gov
} 
protected by water-cooled tungsten-carbide balls; open mercury jet target $(\mathrm{R}=5$ $\mathrm{mm})$ tilted at $100 \mathrm{mrad}$ with a $2 \lambda(\sim 30 \mathrm{~cm})$ beam-jet interaction region; proton beam at $67 \mathrm{mrad}$ with an RMS beam spot size of $1.5 \mathrm{~mm}$; pion/muon yields $\mathrm{Y}$ at the end of the decay channel growing with the beam energy $E_{p}$, while $Y / E_{p}$ having a broad maximum at $\mathrm{E}_{\mathrm{p}} \sim 6 \mathrm{GeV}$.

\section{MARS Event Generators}

The MARS code [3] was and still is the main tool to study the NFMC targetry issues listed above. Particle production in the code is modeled with the built-in event generators. In the current version, MARS15, as a default, an inclusive phenomenological model is used from 3-5 GeV to tens of TeV supplied at lower energies with an exclusive Cascade-Exciton Model code CEM03 [4], combined with the Fermi break-up model, coalescence model, and Generalized Evaporation-fission Model (GEM2). A recent multi-fragmentation extension is available. Alternatively, the Los Alamos Quark-Gluon String Model code, LAQGSM [4], is used for photon, hadron and heavy-ion projectiles from a few $\mathrm{MeV} / \mathrm{A}$ to about a few TeV/A. It shares with CEM03 some modules at E < 3-5 $\mathrm{GeV}$. This option provides a power of full theoretically consistent modeling of exclusive and inclusive distributions of secondary particles, spallation, fission, and fragmentation products. It includes recently improved unified algorithms at intermediate (2 to $10 \mathrm{GeV}$ ) and low $(<20 \mathrm{MeV})$ projectile energies. Among other things, the code has been switched to $\Delta$ and $\mathrm{N}^{*}$ resonance production and interaction at $\mathrm{E}<4.5 \mathrm{GeV}$ with pions produced in $\Delta$-decays. Results of modeling are in an agreement with pion production data, specifically at energies $2<\mathrm{E}<$ $15 \mathrm{GeV}$ (see, for example, recently published papers on BNL-E910 and HARP [5] experiments).

\section{Front-End Channel Acceptance and Pion Production}

The MARS calculated acceptance of the front-end channel described above (see also Ref. [1, 2]) is shown in Fig. 1. It is defined as the probability that a pion of a given angle and momentum at the downstream end of the target yields a surviving muon (or pion) at the end of the channel $(\mathrm{z}=50 \mathrm{~m})$. To be accepted and efficiently captured by the downstream RF channel, the muon must have a kinetic energy in the range $40<\mathrm{T}_{\mu}<180 \mathrm{MeV}$. One sees that the majority of the useful pions has the momenta $0.075<\mathrm{p}<0.475 \mathrm{GeV} / \mathrm{c}$ and is produced in the forward direction $(\theta<\pi / 2)$. For protons interacting with a mercury nucleus (thin target), a pion (kaon) yield $\mathrm{Y}$ in this phase space grows rapidly with proton energy starting from the threshold $(\sim 0.2 \mathrm{GeV})$ to about $2 \mathrm{GeV}$, slowing down at higher energies and reaching a logarithmic growing mode at about $15 \mathrm{GeV}$. As a result, the yield over proton energy, $\mathrm{Y} / \mathrm{E}_{\mathrm{p}}$, has a maximum at $\sim 2 \mathrm{GeV}$ (Fig. 2). 


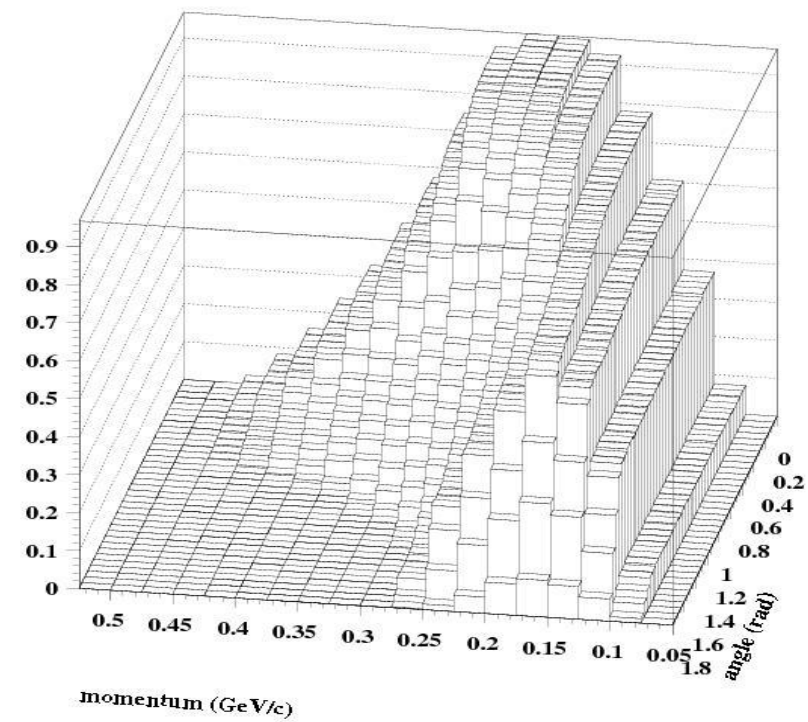

Figure 1. Front-end channel acceptance.

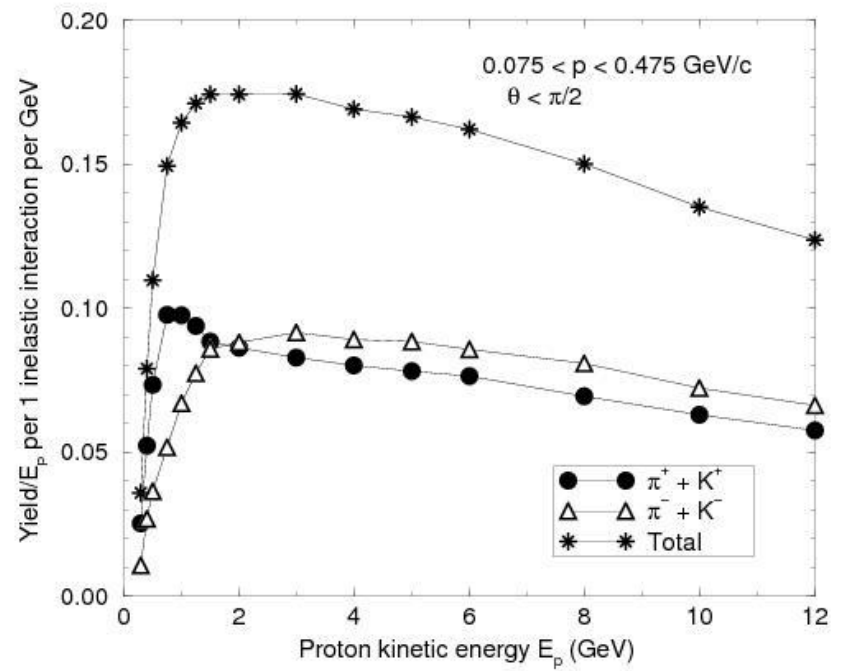

Figure 2. Pion/kaon yield $\mathrm{Y} / \mathrm{E}_{\mathrm{p}}$ for $\mathrm{p}+\mathrm{Hg}$ interactions as calculated with MARS15(LAQGSM). 


\section{Pion Production and Optimal Beam Energy for NFMC}

In a realistic thick target, absorption of low-energy pions in target material and secondary interactions (showering) at high energies move the maximum of $\mathrm{Y} / \mathrm{E}_{\mathrm{p}}$ to a larger $E_{p}$. As a result, the $Y / E_{p}\left(E_{p}\right)$ dependence becomes pretty flat at $2<E_{p}$ $<15 \mathrm{GeV}$. The total acceptance-weighted yield $\mathrm{Y} / \mathrm{E}_{\mathrm{p}}$, modeled with MARS15(LAQGSM) for a $2 \lambda$ thick mercury target, is constant $0.15 \pm 5 \%$ in this energy range with a broad maximum around $\mathrm{E}_{\mathrm{p}}=7 \mathrm{GeV}$. These results are shown in Fig. 3 in comparison with HARP data for a thin tantalum target [5]. The later are acceptance-weighted and corrected for the unmeasured angular region, $\theta<$ $350 \mathrm{mrad}$ (via quadratic extrapolation to $\theta=0$ of HARP group data, $12-19 \%$ effect), and MARS-calculated thick target effect.

One can conclude that any beam energy in the 4-12 GeV range represents a good choice for the proton driver for a neutrino factory or muon collider.

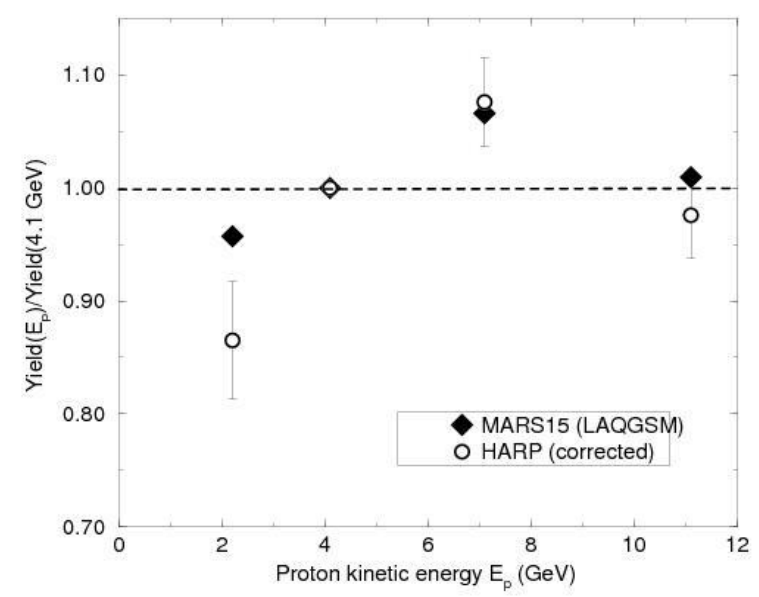

Figure 3. Beam-power normalized muon yield at $\mathrm{z}=50 \mathrm{~m}$ with respect to that at $\mathrm{E}_{\mathrm{p}}=4.1 \mathrm{GeV}$ as calculated with MARS15(LAQGSM) for mercury and measured in Ref. [5] for tantalum.

\section{References}

1. N.V. Mokhov, Nucl. Instr. Meth. Phys. Res., A472, 546 (2001).

2. N.V. Mokhov, Proc. 2001 Part. Accel. Conf., 745-747, Chicago, June 2001.

3. N.V. Mokhov, Fermilab-FN-628 (1995); N.V. Mokhov, S.I. Striganov, in Proc. of Hadronic Shower Simulation Workshop, Fermilab, AIP Conf. Proc., 896, pp. 50-60 (2007); http://www-ap.fnal.gov/MARS/.

4. S.G. Mashnik et al., LANL Report LA-UR-08-2931 (2008); arXiv:0805.0751v1 [nucl th] 6 May 2008.

5. M. G. Catanesi et al. Phys. Rev. C 77, 055207 (2008). 